\title{
Experimental study on retardation of a heavy NAPL vapor in partially saturated porous media
}

\author{
Simon Matthias Kleinknecht ${ }^{1}$, Holger Class ${ }^{2}$, and Jürgen Braun ${ }^{1}$ \\ ${ }^{1}$ Facility for Subsurface Remediation, Institute for Modelling Hydraulic and Environmental Systems, University of Stuttgart, \\ Pfaffendwaldring 61, 70569 Stuttgart, Germany \\ ${ }^{2}$ Department of Hydromechanics and Modelling of Hydrosystems, Institute for Modelling Hydraulic and Environmental \\ Systems, University of Stuttgart, Pfaffendwaldring 61, 70569 Stuttgart, Germany \\ Correspondence to: Simon Matthias Kleinknecht (simon.kleinknecht@iws.uni-stuttgart.de)
}

Received: 25 August 2016 - Discussion started: 30 August 2016

Revised: 12 January 2017 - Accepted: 13 February 2017 - Published: 8 March 2017

\begin{abstract}
Non-aqueous-phase liquid (NAPL) contaminants introduced into the unsaturated zone spread as a liquid phase; however, they can also vaporize and migrate in a gaseous state. Vapor plumes migrate easily and thus pose a potential threat to underlying aquifers. Large-scale column experiments were performed to quantify partitioning processes responsible for the retardation of carbon disulfide $\left(\mathrm{CS}_{2}\right)$ vapor in partially saturated porous media. The results were compared with a theoretical approach taking into account the partitioning into the aqueous phase as well as adsorption to the solid matrix and to the air-water interface. The experiments were conducted in large, vertical columns (i.d. of $0.109 \mathrm{~m}$ ) of $2 \mathrm{~m}$ length packed with different porous media. A slug of $\mathrm{CS}_{2}$ vapor and the conservative tracer argon was injected at the bottom of the column followed by a nitrogen chase. Different seepage velocities were applied to characterize the transport and to evaluate their impact on retardation. Concentrations of $\mathrm{CS}_{2}$ and argon were measured at the top outlet of the column using two gas chromatographs. The temporal-moment analysis for step input was employed to evaluate concentration breakthrough curves and to quantify dispersion and retardation. The experiments conducted showed a pronounced retardation of $\mathrm{CS}_{2}$ in moist porous media which increased with water saturation. The comparison with an analytical solution helped to identify the relative contributions of partitioning processes to retardation. Thus, the experiments demonstrated that migrating $\mathrm{CS}_{2}$ vapor is retarded as a result of partitioning processes. Moreover, $\mathrm{CS}_{2}$ dissolved in the bulk water is amenable to biodegradation. The first evidence of $\mathrm{CS}_{2}$ decay by biodegradation was found in the experiments. The find-
\end{abstract}

ings contribute to the understanding of vapor-plume transport in the unsaturated zone and provide valuable experimental data for the transfer to field-like conditions.

\section{Introduction}

Subsurface contamination is a major concern in industrialized as well as in developing and emerging countries. Nonaqueous-phase liquid (NAPL) contaminants introduced into the unsaturated zone spread as a liquid phase; however, they can also vaporize and migrate in a gaseous state. In particular, vapor (gas) plumes migrate easily in the unsaturated zone (Barber and Davis, 1991; Davis et al., 2005, 2009; Höhener et al., 2006; Brusseau et al., 2013). When assessing the danger of groundwater contamination by downwardmigrating vapor plumes, retention effects on transport are of major interest. Processes such as dissolution into the bulk water or adsorption at interfaces affect the migration of vapors in the unsaturated zone. Vapor retardation could potentially slow down migration and reduce the total contaminant mass eventually reaching, and thus endangering, the groundwater. While fate and transport of vapor plumes have attracted a great deal of attention over the past years, further (contaminant-specific) investigations are necessary to improve the process understanding required to assess the threat to the environment (Rivett et al., 2011).

Carbon disulfide $\left(\mathrm{CS}_{2}\right)$ is a toxic, industrial, non-polar (among others) solvent used to manufacture viscose rayon. It is a dense non-aqueous-phase liquid and highly volatile 
at standard conditions. Its vapor is characterized by a high density (1.6 times the air density) and is explosive. Hence, $\mathrm{CS}_{2}$ vapor can migrate in the unsaturated zone, intrude into buildings, and distant ignition is possible. The US Agency for Toxic Substances and Disease Registry ranks $\mathrm{CS}_{2}$ 168th on the Substance Priority List (SPL) of hazardous substances found on US EPA National Priority List (NPL) sites (US Agency for Toxic Substances and Disease Registry, 2015). $\mathrm{CS}_{2}$ has been found in 122 of the 1844 sites on the NPL (US Environmental Protection Agency, 2016). In addition, $\mathrm{CS}_{2}$ has also been detected on $\mathrm{CCl}_{4}$-contaminated sites as a result of abiotic degradation of $\mathrm{CCl}_{4}$ (Davis et al., 2003).

Experimental studies (e.g., Brusseau et al., 1997; Kim et al., 1998; Costanza-Robinson et al., 2013) have been conducted to investigate retardation of the most common volatile organic carbon (VOC) in unsaturated porous media using short columns in the range of few decimeters and high flow velocities. Experimental results have been compared with standard as well as advanced advection-dispersion models (Popovičová and Brusseau, 1998; Toride et al., 2003). Corley et al. (1996) showed that low concentrations of volatile organic compounds distribute in the bulk phases (air, water, and solid), adsorb to the air-water interface, and partition into intraparticle pores in unsaturated and saturated porous media. While it has been demonstrated in experiments that the gas-water interface poses a high potential for retardation (Brusseau et al., 1997; Costanza-Robinson and Brusseau, 2002b), determining the effective interfacial area, which controls the contaminant mass transfer, is still subject of intensive research (Goss, 2009; Kibbey and Chen, 2012; Brusseau et al., 2015). Mayes et al. (2003) stated that immobile water in pores could act as a short-term sink and as a long-term source of potential contaminants. The effect of moisture content on vapor retention has also been described by Cabbar and Bostanci (2001) and Maxfield et al. (2005), who discovered retardation to be negatively correlated to water saturation due to preferred adsorption on the solid matrix of certain components. The latter has additionally shown the dependency of retardation on the properties of the chemical component of interest. For instance, noble gases show no retardation at all. In summary, retardation of a contaminant vapor is a combination of different partitioning processes; their relative contribution depends on the physicochemical properties of the component as well as the properties of the porous medium (Costanza-Robinson et al., 2013).

The component- and porous-medium-dependent behavior of gas-phase retention emphasizes the need for a thorough investigation into retardation of carbon disulfide $\left(\mathrm{CS}_{2}\right)$ in partially saturated porous media. No detailed experimental studies on the retardation behavior of $\mathrm{CS}_{2}$ vapor are available. Therefore, the first objective of this study was to design and conduct a large-scale column experiment to quantify retardation of $\mathrm{CS}_{2}$ with clearly defined and controlled boundary conditions. The experiments were conducted in vertical columns (i.d. of $0.109 \mathrm{~m}$ ) of $2 \mathrm{~m}$ length packed with a porous medium. They were carried out under dry conditions and at static water saturations. Reproducible water saturations (initial conditions) were obtained by saturation with water and subsequent drainage under controlled conditions at predetermined capillary pressures. Tensiometers installed along the column were used to derive water-saturation profiles. A slug of $\mathrm{CS}_{2}$ vapor and a non-retarding, conservative tracer (argon) was injected via an injection section at the bottom of the column. Velocities were varied in the range 25 to $200 \mathrm{~cm} \mathrm{~h}^{-1}$ in different experiments. Effluent concentrations of $\mathrm{CS}_{2}$ and argon were measured online with gas chromatographs at the top outlet of the column. This setup enabled for the quantification of $\mathrm{CS}_{2}$ retardation as a function of porous medium, water saturation, and velocity. The second objective was to compare the experimental results with a theoretical approach, taking into account the different partitioning domains available for $\mathrm{CS}_{2}$ and eventually responsible for retardation. This allowed to quantify contributions of the partitioning processes to total retardation. Hence, fundamental knowledge regarding the potential to delay or prevent a contamination of an underlying aquifer was gained.

\section{Materials and methods}

\subsection{Experimental setup}

The experiments were conducted in vertical, stainless steel columns of $2 \mathrm{~m}$ length packed with two different types of porous media (Fig. 1). The column (length of $2 \mathrm{~m}$, i.d. of $0.109 \mathrm{~m}$ ) consisted of two custom-built $1 \mathrm{~m}$ long sections.

The ports along the column at a distance of $25 \mathrm{~cm}$ allowed for the installation of tensiometers to monitor capillary pressures. At the bottom of the column, the injection section with a base plate was installed. Into this base plate, a porous plate made of recrystallized silicon carbide was glued to act as a suction plate for the water drainage. The bottom of the column was realized as a constant-mass-flux boundary while the top was open to the surroundings, hence, at constant pressure.

Two different types of porous media (Table 1) were used in the experiments: fine glass beads (soda-lime glass, Sigmund Lindner, Warmensteinach, Germany) and Geba fine sand (Quarzsande $\mathrm{GmbH}$, Eferding, Austria).

Their grain-size distributions as well as capillary pressurewater saturation relationships are shown in Fig. 2. Figure 3 shows scanning electron microscopy images of both materials illustrating their different shape and surface roughness.

The columns were packed by dry pluviation using a sand rainer (Rad and Tumay, 1987; Lagioia et al., 2006). This ensured a homogeneous porous medium throughout. The columns were sealed with cover plates equipped with $1 / 8^{\prime \prime}$ tube fittings (SS-6M0-1-4RT, Swagelok). The experiments were carried out under dry conditions as well as at residual, static water saturation. Comparable initial conditions for each experiment were guaranteed by a setup 


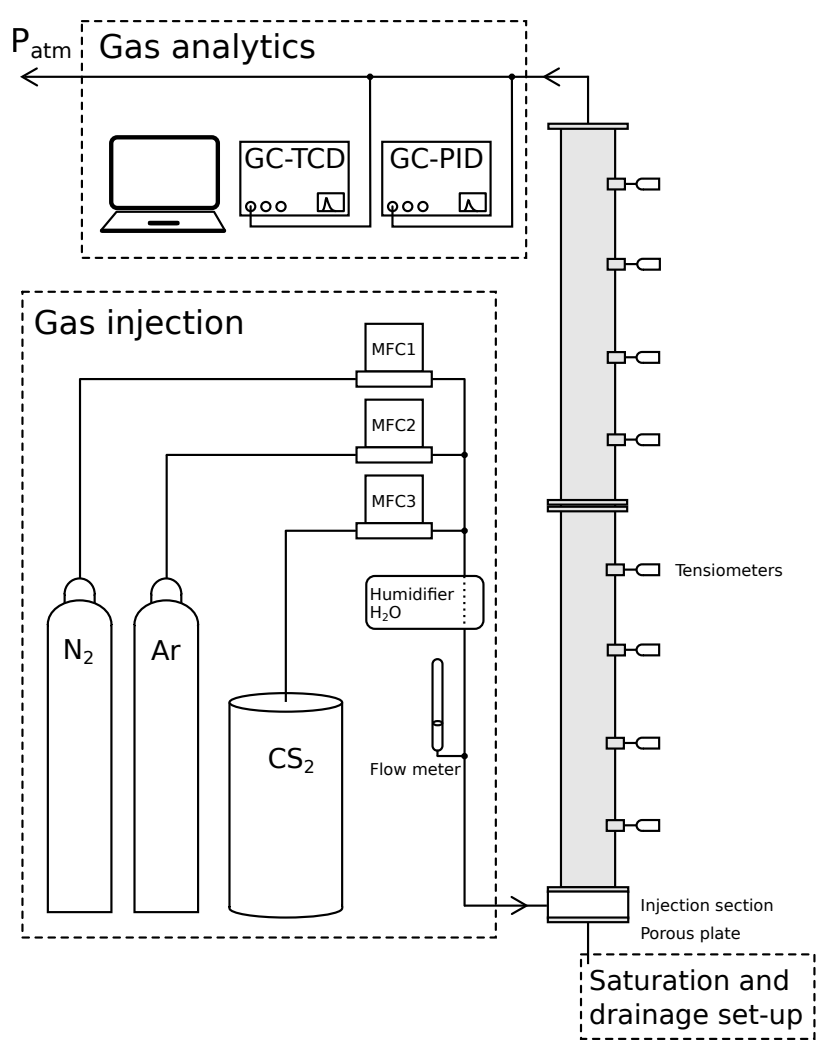

Figure 1. Flowchart of the vapor-retardation experiment showing column, injection, and saturation/drainage setup.

controlling and monitoring saturation and drainage. The drainage of the porous media was realized by means of the porous plate at the bottom of the column exclusively permeable for water when fully saturated. This allowed for setting the water table lower than the bottom of the column or flume. The saturations followed the capillary pressure-saturation relationship (Fig. 2) measured in the laboratory. The water saturation was monitored using the tensiometers installed at the column ports. The tensiometer used for measuring capillary pressures consisted of a ceramic frit (length of $8 \mathrm{~mm}$, o.d. of $6.5 \mathrm{~mm}$, pore size of $2.5 \mu \mathrm{m}$, porosity of $45 \%$; porous ceramics, Soilmoisture Equipment Corp., Santa Barbara, USA) glued in a stainless steel capillary (length of $200 \mathrm{~mm}$, o.d. of $6 \mathrm{~mm}$, i.d. of $4 \mathrm{~mm}$ ). In addition, the column was placed on a scale to permanently monitor its weight and thus the total amount of water.

The injection section at the bottom of the column allowed for the injection of a gas-mixture slug at a predefined mass flux and, in addition, for a controlled upward flow stabilizing the vapor front. The $\mathrm{CS}_{2}$ vapor was prepared prior to injection.

Table 2 shows the physicochemical properties of the contaminant carbon disulfide $\left(\mathrm{CS}_{2}\right)$ at $20^{\circ} \mathrm{C}$ and $1013.15 \mathrm{hPa}$. A predefined amount of liquid $\mathrm{CS}_{2}$ was injected into a barrel $(V=50 \mathrm{~L})$ and pressurized to an excess pressure of
Table 1. Characteristic properties of the porous media used for the experiments.

\begin{tabular}{|c|c|c|}
\hline Parameter & $\begin{array}{r}\text { Fine glass } \\
\text { beads }\end{array}$ & $\begin{array}{r}\text { Geba fine } \\
\text { sand }\end{array}$ \\
\hline Bulk density, $\mathrm{kg} \mathrm{m}^{-3}$ & 1420 & 1390 \\
\hline Grain size, $\mathrm{mm}$ & $0.1-0.2$ & $0.06-0.35$ \\
\hline Permeability, $\mathrm{m}^{2}$ & $1 \times 10^{-11}$ & $1 \times 10^{-11}$ \\
\hline Grain diameter $d_{50}, \mu \mathrm{m}$ & 162 & 140 \\
\hline Pore diameter (median), $\mu \mathrm{m}$ & 66 & 39 \\
\hline Smooth-sphere assumption SA, $\mathrm{cm}^{-1}$ & 22.8 & 24.3 \\
\hline \multicolumn{3}{|l|}{ van Genuchten (constrained) } \\
\hline$\alpha, \mathrm{cm}^{-1}$ & 0.0193 & 0.0145 \\
\hline$n$, dimensionless & 17.783 & 10.305 \\
\hline$\theta_{\mathrm{s}}, \mathrm{cm}^{3} \mathrm{~cm}^{-3}$ & 0.392 & 0.460 \\
\hline$\theta_{\mathrm{r}}, \mathrm{cm}^{3} \mathrm{~cm}^{-3}$ & 0.043 & 0.071 \\
\hline
\end{tabular}

about 2 bar with nitrogen to ensure defined vapor properties. The tracer argon was provided from a gas cylinder (Westfalen AG, Münster, Germany). Constant mass fluxes of the injected $\mathrm{CS}_{2}$ vapor and of the conservative tracer argon were critical to the experiment. Mass fluxes of argon, $\mathrm{CS}_{2}$ vapor, and nitrogen were controlled by mass-flow controllers (ELFLOW, $Q_{\max }=3,50$, and $10 \mathrm{~mL} \mathrm{~m}^{-1}$; Bronkhorst HighTech B.V., Ruurlo, the Netherlands). Complete gas tightness of the entire setup was ensured by using $1 / 8^{\prime \prime}$ stainless steel capillaries throughout. The slug of the gas mixture $\left(\mathrm{CS}_{2}\right.$, argon $\left(Q_{\text {Ar }} / Q_{\text {total }}=1.4 \%\right)$, and carrier nitrogen $)$ was injected and then pushed through the column using a nitrogen chase at the same flow rate. The nitrogen chase allowed to observe the contaminant recovery and the reversibility of partitioning processes.

In the case of moist experiments, the gas-mixture slug was humidified with ultra-pure water (RH of $100 \%$ ) to avoid a drying up of the moist porous medium. For the preparation of the gas-mixture slug, a custom-built miniature vaporizer (ICTV; University of Stuttgart, Germany) with an ultralow volume pump (M6; VICI AG International, Schenkon, Switzerland) was used. The nitrogen used for the chase was bubbled through a gas scrubber filled with ultra-pure water. The inlet steel capillary loop (length of $4 \mathrm{~m}$ ) and the scrubber were placed in a temperature-controlled water bath (Ministat 125, Huber Kältemaschinenbau $\mathrm{GmbH}$, Germany) to minimize temperature-induced fluctuations during the experiments. The mass balance was closed based on the flow rate measured at the inflow, and the injection and effluent concentrations.

In the column outflow, $\mathrm{CS}_{2}$ and argon concentration were measured to quantify retardation in dry and moist porous media. Two gas chromatographs were directly connected in line to the column outlet. $\mathrm{CS}_{2}$ concentrations were determined using a gas chromatograph with a photoionization detector (GC-PID HE1, Meta Messtechnische Systeme GmbH, Dres- 
Table 2. Physicochemical properties of contaminant carbon disulfide $\left(\mathrm{CS}_{2}\right)$ at $20^{\circ} \mathrm{C}$ and $1013.15 \mathrm{hPa}$. CAS indicates Chemical Abstracts Service.

\begin{tabular}{lrl}
\hline Parameter & Value & Reference \\
\hline CAS number & $75-15-0$ & \\
Molecular weight $\left(M_{\mathrm{CS} 2}\right), \mathrm{g} \mathrm{mol}^{-1}$ & 76.1 & Budavari (1996) \\
Density of liquid $(\rho), \mathrm{kg} \mathrm{m}^{-1}$ & 1263 & Budavari (1996) \\
Solubility in water $\left(c_{\mathrm{W}, \mathrm{sat}}\right), \mathrm{g} \mathrm{L}^{-1}$ & 2.1 & Riddick et al. (1986) \\
Henry constant $\left(H_{\mathrm{cc}}\right)$, dimensionless & 1.04 & Lide (2005) \\
Octanol-water partitioning coefficient $\left(\log \left(K_{\mathrm{OW}}\right)\right)$, dimensionless $^{-1}$ & 2.00 & US Environmental Protection Agency (1996) \\
Organic-carbon partitioning coefficient $\left(K_{O C}\right), \mathrm{L} \mathrm{kg}^{-1}$ & 45.7 & US Environmental Protection Agency (1996) \\
Air-water partitioning coefficient $\left(K_{\mathrm{IA}}\right), \mathrm{cm}$ & $6.87 \times 10^{-6}$ & Valsaraj (1988) \\
Boiling point $\left(T_{\mathrm{B}}\right),{ }^{\circ} \mathrm{C}$ & 46.5 & Budavari (1996) \\
Vapor pressure $\left(P_{\mathrm{sat}}\right), \mathrm{hPa}$ & 396.9 & Wagner equation \\
Saturation concentration in gas phase $\left(c_{\mathrm{a}, \mathrm{sat}}\right), \mathrm{kg} \mathrm{m}^{-3}$ & 1.239 & Ideal gas law \\
Diffusion coefficient in air $\left(D_{\mathrm{CS}}\right.$ Air $), \mathrm{cm}^{2} \mathrm{~s}^{-1}$ & $9.71 \times 10^{-2}$ & Chapman-Enskog \\
\hline
\end{tabular}
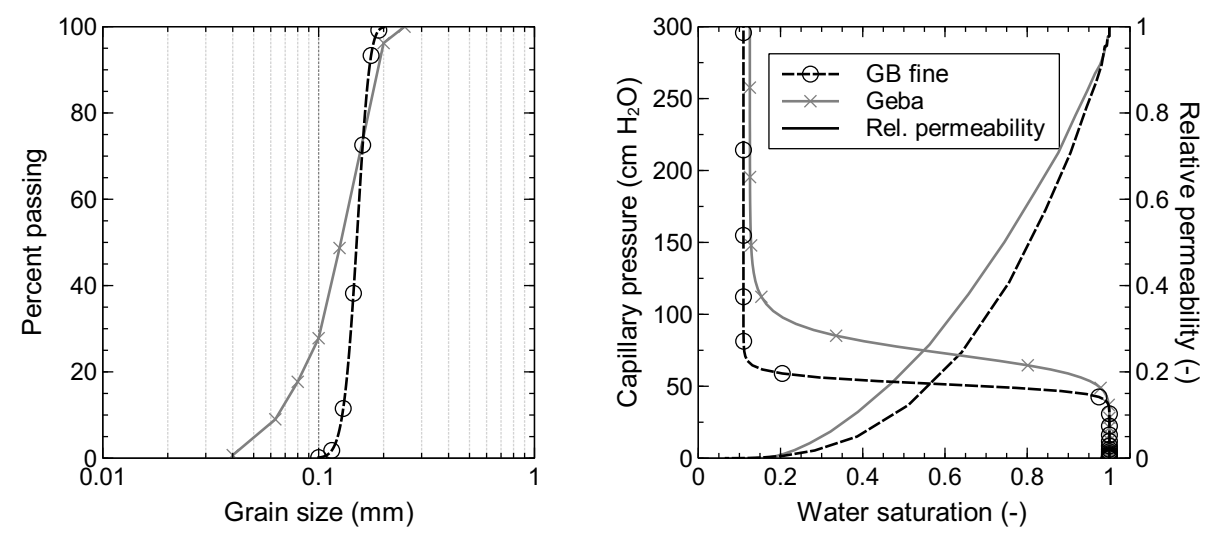

Figure 2. Grains-size distribution, capillary pressure-saturation relationship, and relative permeabilities (for the wetting phase) of materials used in experiments.
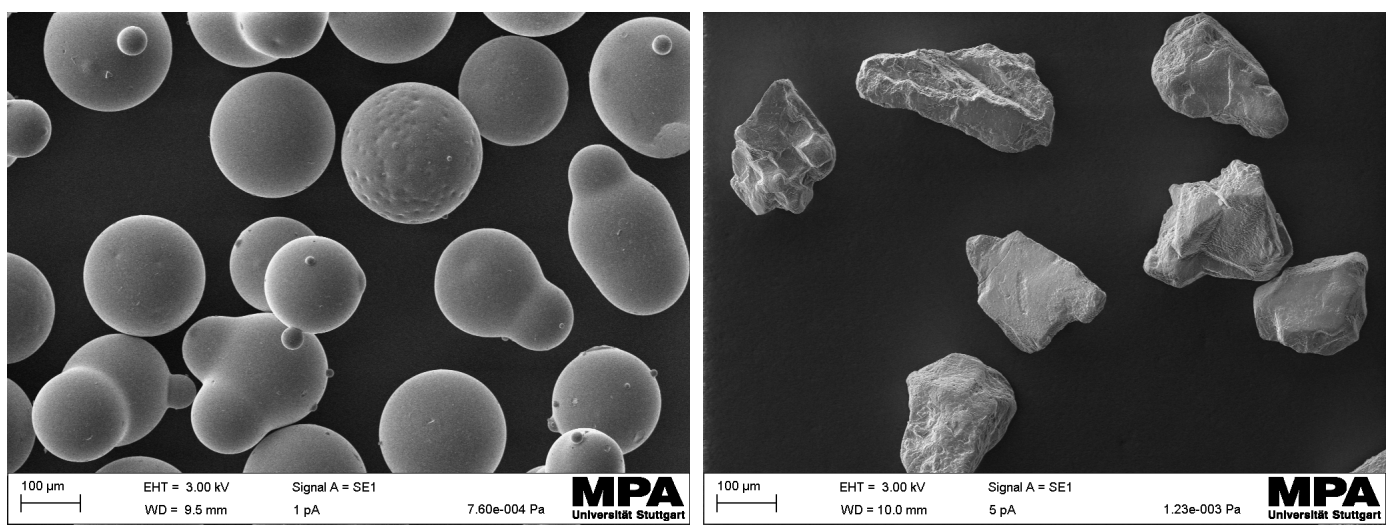

Figure 3. Scanning electron microscopy images of fine glass beads (left panel) and Geba fine sand (right panel) at a scale of $100 \mu \mathrm{m}$.

den, Germany) and argon concentrations were determined using a gas chromatograph with thermal conductivity detector (GC-TCD, multiple gas analyzer 8610-0270, SRI Instruments Europe GmbH, Bad Honnef, Germany). Single-point calibrations were conducted prior to and after each run. Mea- surement intervals were set depending on the flow velocity such that a high temporal resolution $(0.021$ to $0.065 \mathrm{PV})$ of the breakthrough curves was obtained. Prior to the start of the slug injection, the concentration of $\mathrm{CS}_{2}$ and argon in the slug mixture was measured as a base to normalize concen- 
trations. A relative pressure transducer connected to the column inlet before the injection section was used to monitor the injection pressure. Since the top column outlet was open to the atmosphere $\left(P_{\mathrm{atm}}\right)$, this corresponded to the pressure loss caused by the flow through the porous medium. Temperature sensors and absolute pressure transducers continuously measured and recorded ambient and water bath temperature as well as atmospheric pressure in the vicinity of the experiment.

\subsection{Experimental procedure}

Various experiment series were conducted in two different porous media (fine glass beads and Geba fine sand) under both dry and partially saturated (moist) conditions. Within each series, the columns were not repacked and no saturation-and-drainage cycle (SD) was carried out since first tests proved that the partitioning processes were fully reversible. The water saturation or total amount of water was monitored throughout the experiment. The slug of the gas mixture was injected with a predefined mass flux into the bottom of the $2 \mathrm{~m}$ long column such that it resulted in the designated seepage velocity. In each series, experiments were performed with different velocities including 25, 50, 100, and $200 \mathrm{~cm} \mathrm{~h}^{-1}$ (approximately $0.125,0.25,0.5$, and $1.0 \mathrm{PV} \mathrm{h}^{-1}$ ) to observe kinetic effects on retardation. A slug of about 3.5 PV was used which corresponded to injection durations of approximately $3.5,7,14$, and $28 \mathrm{~h}$ depending on the respective velocities. This ensured a residence time (plus safety factor) sufficient to attain steady-state conditions and for partitioning processes to reach equilibrium.

The experiments were conducted in four steps. In the first step, the flow rates (slug and chase) were adjusted to match the target seepage velocity. In the second step, the column was flushed with nitrogen. While maintaining constant flux, the inflow was switched to the slug injection of the gas mixture in the third step. After injecting 3.5 PV, it was switched back to the nitrogen chase (fourth step).

\subsection{Data evaluation}

The quantification of retardation was based on gas concentration measurements of $\mathrm{CS}_{2}$ and argon. Possible influences on the determined retardation factors due to experimental artifacts, such as a deviation between theoretical and actual gas-effective pore volume, had to be taken into consideration. Hence, for each experiment, the breakthrough curve of $\mathrm{CS}_{2}$ was related to that of argon. Concentrations were normalized with respect to the steady-state concentrations ( $\left.c=c_{\exp } / c_{\mathrm{ss}}\right)$. Mass balance was calculated from concentration data and measured gas flow rates. Data were evaluated based on elapsed time and then correlated via flow rate, resulting from mass flux, to gas-effective pore volume. Breakthrough curves were evaluated using the temporal-moment analysis (TMA) for a step input (slug) as proposed by $\mathrm{Yu}$ et al. (1999) and Luo et al. (2006). The advantage of TMA "is that no underlying physical model is needed for calculating the travel times" (Yu et al., 1999, p. 3571), and the breakthrough curves of the $\mathrm{CS}_{2}-\mathrm{Ar}$ mixture (slug front) as well as the nitrogen chase (slug tail) can be evaluated individually.

TMA was applied to obtain transport parameters (seepage velocity, Eq. A9; dispersion coefficient, Eq. A10) and mean breakthrough arrival time (Eq. A7) from concentration breakthrough curves. The retardation factor $R$ of $\mathrm{CS}_{2}$ vapor was calculated from the ratio of the respective moments or mean breakthrough arrival time.

$R=\frac{\tau_{\mathrm{CS}_{2}}}{\tau_{\mathrm{Ar}}}=\frac{M_{1, \mathrm{CS}_{2}}}{M_{1, \mathrm{Ar}}}$

This ensured the independence from experimentally induced deviations and thus allowed to delineate the impact of water saturation and velocity on retardation.

Retardation of a component can be estimated using analytical solutions based on experimental parameters and component-dependent coefficients for the different partitioning domains. Brusseau et al. (1997) showed that the theoretical retardation coefficient of a gaseous component in the unsaturated zone can be calculated with

$R_{\mathrm{t}}=1+\beta_{\mathrm{w}}+\beta_{\mathrm{s}}+\beta_{\mathrm{IA}}=1+\frac{\theta_{\mathrm{w}}}{\theta_{\mathrm{a}} K_{\mathrm{H}}}+\frac{\rho_{\mathrm{b}} K_{\mathrm{D}}}{\theta_{\mathrm{a}} K_{\mathrm{H}}}+\frac{K_{\mathrm{IA}} A_{\mathrm{IA}}}{\theta_{\mathrm{a}}}$,

where $\theta_{\mathrm{W}}$ is volumetric water content, $\theta_{\mathrm{a}}$ is gas-filled porosity, $K_{\mathrm{H}}$ (dimensionless) is the Henry's constant, $K_{\mathrm{D}}$ $\left(\mathrm{cm}^{3} \mathrm{~g}^{-1}\right)$ is the sorption coefficient for water-saturated conditions, $\rho_{\mathrm{b}}\left(\mathrm{g} \mathrm{cm}^{-3}\right)$ is the dry soil bulk density, $K_{\mathrm{IA}}(\mathrm{cm})$ is the adsorption coefficient at the gas-water interface, and $A_{\text {IA }}\left(\mathrm{cm}^{2} \mathrm{~cm}^{-3}\right)$ is the specific surface area of the gas-water interface.

The coefficient $K_{\mathrm{D}}$ for $\mathrm{CS}_{2}$ can be estimated with

$K_{\mathrm{D}}=K_{\mathrm{OC}} \times f_{\mathrm{OC}}$,

where $K_{\mathrm{OC}}\left(\mathrm{L} \mathrm{kg}^{-1}\right)$ is the soil organic carbon partitioning coefficient and $f_{\mathrm{OC}}$ is the fraction of organic carbon in the soil material. The air-water partitioning coefficient $K_{\text {IA }}$ of $\mathrm{CS}_{2}$ can be estimated using empirical correlations between the interfacial-water partitioning constant $K_{\text {IW }}$ and the octanol-water partitioning coefficient $K_{\text {OW }}$ (Valsaraj, 1988).

$K_{\mathrm{IW}}=3 \times 10^{-7} K_{\mathrm{OW}}^{0.68}$
$K_{\mathrm{IA}}=\frac{K_{\mathrm{IW}}}{K_{\mathrm{H}}}$

The air-water interfacial area can be estimated with a correlation found by Costanza-Robinson et al. (2008) based on Xray microtomography measurements of glass beads and natural sands.

$A_{\mathrm{IA}}=\mathrm{SA}\left[(-0.9112) S_{\mathrm{W}}+0.9031\right]$,

where $\mathrm{SA}$ is the geometric surface area according to the smooth-sphere assumption (Table 1) and $S_{\mathrm{W}}$ is the water saturation. 
Table 3. Experimental conditions of vapor-retardation experiments in fine glass beads and Geba fine sand in dry and moist conditions (series).

\begin{tabular}{lrrrr}
\hline Series & 1 & 2 & 3 & 4 \\
Condition & dry & moist & moist & moist \\
\hline & \multicolumn{4}{c}{ Fine glass beads } \\
\hline Porosity $(\phi)$ & 0.40 & 0.40 & 0.40 & 0.40 \\
Mean water saturation $\left(S_{\mathrm{W}}\right)$ & 0.0 & 0.088 & 0.154 & 0.073 \\
Eff. pore volume, $L$ & 7.72 & 7.04 & 6.53 & 7.16 \\
\hline & \multicolumn{5}{c}{ Geba fine sand } \\
\hline Porosity $(\phi)$ & 0.40 & 0.40 & 0.40 & - \\
Mean water saturation $\left(S_{\mathrm{W}}\right)$ & 0.0 & 0.162 & 0.150 & - \\
Eff. pore volume, $L$ & 7.58 & 6.35 & 6.45 & - \\
\hline
\end{tabular}

\section{Results and discussion}

Column experiments were conducted with dry and moist porous media to characterize retardation of $\mathrm{CS}_{2}$ vapor. Table 3 shows the experimental conditions of each series performed in fine glass beads and Geba fine sand.

Several series of experiments were performed in each porous medium to quantify retardation. Series 1 refers to the experiments conducted in dry porous media while Series 2 to 4 refer to the experiments in moist conditions. A saturation-and-drainage cycle was performed prior to each moist series. A slug of 3.5 PV of the gas mixture was injected ensuring, even for high flow rates, a sufficient residence time to reach equilibrium in the $2 \mathrm{~m}$ long column. Different velocities $\left(25,50,100\right.$, and $\left.200 \mathrm{~cm} \mathrm{~h}^{-1}\right)$ were applied, based on previously conducted experiments investigating density-driven vapor migration (Kleinknecht et al., 2015). Breakthrough curves under the prevailing experimental conditions were determined from concentration measurements at the column outlet. The temporal-moment analysis (Sect. 2.3) was applied to the breakthrough curves (BTCs) to quantify diffusion/dispersion and retardation as a function of the porous media, the water saturation, and the flow conditions. A detailed summary of all experiments (experimental conditions, injected mass, and mass recovery) is given in Tables B1 and B2 in the Appendix.

\subsection{Water saturations}

The moist porous medium required for this investigation was obtained by saturation and subsequent drainage via a suction plate. The capillary pressure was measured with tensiometers installed at the column ports to derive water saturations along the column $\left(P_{\mathrm{c}}-S_{\mathrm{w}}\right.$, Fig. 2). Mean water saturations of the moist series are given in Table 3 .

Figure 4 shows the initial water-saturation profiles along the column measured in fine glass beads (only Series 4) and Geba fine sand (Series 2 and 3).

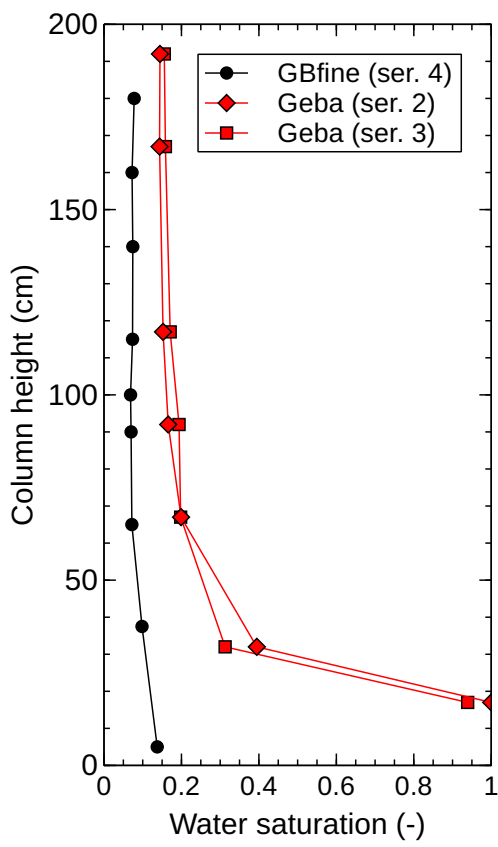

Figure 4. Initial water saturation.

Unfortunately, no tensiometer measurement data were available for Series 2 and 3 in fine glass beads. Thus, the missing profiles have to be considered an element of uncertainty when evaluating retardation of $\mathrm{CS}_{2}$ of these series. However, the available profile of Series 4 revealed a uniform saturation along the column, only slightly increasing toward the bottom from $S_{\mathrm{w}}=0.07$ to 0.14 . The very narrow and uniform grain-size distribution of the fine glass beads was responsible for a sharp transition from full to residual saturation (see $P_{\mathrm{c}}-S_{\mathrm{w}}$ curve in Fig. 2), thus favoring a uniform saturation profile. In Geba fine sand, a constant water saturation of $S_{\mathrm{w}}=0.15$ above a column height of $70 \mathrm{~cm}$ was measured. However, both profiles showed a pronounced increase in the water saturation toward the bottom of the column, apparently reaching fully saturated conditions according to the $P_{\mathrm{c}}-S_{\mathrm{w}}$ relationship of Geba fine sand (Fig. 2). Still, capillary pressures of $P_{\mathrm{c}}=55$ and $65 \mathrm{hPa}$ were measured at the lowest port. The suction applied via the porous plate was limited by its air entry pressure. A further decrease of pressure would have resulted in a breakthrough of air (continuous gas phase). The mean water saturation (Table 3 ) but also the observed profiles were expected to have an impact on the retardation behavior of $\mathrm{CS}_{2}$, as discussed in Sect. 3.3.

A constant water-saturation profile in the porous medium was ensured by the humidification of all gases (gas-mixture slug and nitrogen chase) prior to injection. This was confirmed by a continuous weight measurement of the entire column throughout all experiments conducted within a series. Hence, the initial water-saturation profile could be maintained during the experiments. 


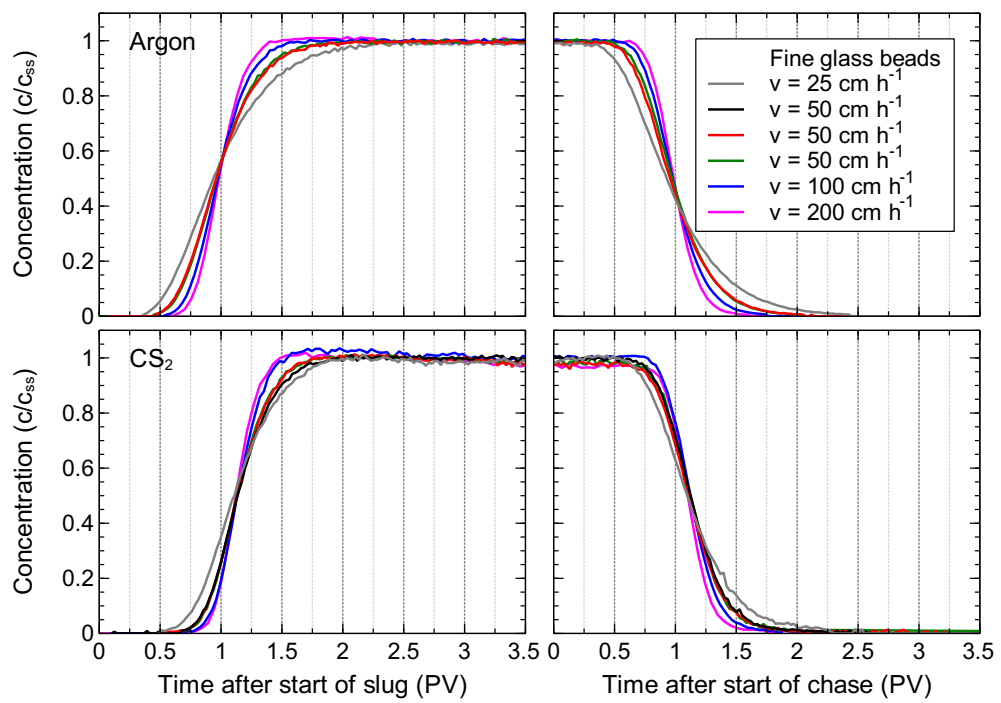

Figure 5. Breakthrough curves of $\mathrm{CS}_{2}$ and $\mathrm{Ar}$ in moist fine glass beads $\left(S_{\mathrm{W}}=0.088\right)$ for different velocities.

\subsection{Impact of velocity on breakthrough}

Different migration velocities were applied to study their impact on the transport of argon and $\mathrm{CS}_{2}$. Figure 5 shows breakthrough curves of $\mathrm{CS}_{2}$ and argon as a function of pore volume for different flow conditions (velocities) in moist fine glass beads. The breakthrough curves were adapted to the actual gas-effective pore volume determined from the mean breakthrough arrival time $\tau_{\mathrm{Ar}}$ (Eq. A7) of the conservative tracer argon. Seepage velocities of about $25,50,100$, and $200 \mathrm{~cm} \mathrm{~h}^{-1}$ (residence time of about $8,4,2$, and $1 \mathrm{~h}$ ) were applied successively in the same column and under similar initial conditions. The lines represent measured concentrations $\left(c / c_{\mathrm{ss}}\right)$ normalized to steady-state concentration. The graphs are split and the right-hand side shows the outflow concentrations after the injection was switched from the gasmixture slug to the nitrogen chase. The skewness of a BTC is a result of the longitudinal molecular diffusion and the mechanical mixing. The BTCs of argon and $\mathrm{CS}_{2}$ shown in the graphs illustrate that the skewness increased with decreasing seepage velocity as a result of diffusion during the longer residence time.

Dispersion coefficients were determined from temporalmoment analysis (see Sect. A3) of the breakthrough curves. Dispersion coefficients of argon and $\mathrm{CS}_{2}$ increased from $D_{\mathrm{Ar}}=0.089$ to $0.142 \mathrm{~cm}^{2} \mathrm{~s}^{-1}$ and $D_{\mathrm{CS}}^{2}=0.033$ to $0.074 \mathrm{~cm}^{2} \mathrm{~s}^{-1}$ as a function of the seepage velocity. Under static conditions $\left(v=0 \mathrm{~cm} \mathrm{~h}^{-1}\right)$, the effective binary diffusion coefficients $D^{*}$ in porous media should apply. The effective binary diffusion coefficients $D^{*}$ and the dispersivity $\alpha$ were determined based on a linear regression fitted to the dispersion coefficients as a function of the velocity for each porous medium. The $y$ intercepts of the regression lines represent the coefficient $D^{*}$ and the slopes express disper-
Table 4. Theoretical and experimental effective binary diffusion coefficient $D^{*}$ of argon and $\mathrm{CS}_{2}$, dispersivity $\alpha$, and coefficient of determination $R^{2}$ of linear regression determined from experiments in moist porous media (Series 2).

\begin{tabular}{|c|c|c|c|}
\hline \multirow{3}{*}{\multicolumn{2}{|c|}{$\begin{array}{l}\text { Porous medium } \\
\text { Water saturation } S_{\mathrm{W}} \\
\text { Tortuosity } \tau\end{array}$}} & Fine glass beads & Geba fine sand \\
\hline & & 0.088 & 0.162 \\
\hline & & 0.220 & 0.161 \\
\hline \multirow{4}{*}{ Argon } & $D_{t}^{*}, \mathrm{~cm}^{2} \mathrm{~s}^{-1}$ & 0.0386 & 0.0284 \\
\hline & $D^{*}, \mathrm{~cm}^{2} \mathrm{~s}^{-1}$ & 0.0909 & 0.0966 \\
\hline & $\alpha, \mathrm{cm}$ & 1.029 & 0.313 \\
\hline & $R^{2}$ (lin. regression) & 0.919 & 0.254 \\
\hline \multirow{4}{*}{$\mathrm{CS}_{2}$} & $D_{t}^{*}, \mathrm{~cm}^{2} \mathrm{~s}^{-1}$ & 0.0213 & 0.0157 \\
\hline & $D^{*}, \mathrm{~cm}^{2} \mathrm{~s}^{-1}$ & 0.0263 & 0.0332 \\
\hline & $\alpha, \mathrm{cm}$ & 0.888 & 0.552 \\
\hline & $R^{2}$ (lin. regression) & 0.987 & 0.952 \\
\hline
\end{tabular}

sivity $\alpha$ of the respective porous medium. The theoretical coefficient $D_{\mathrm{t}}^{*}$ was determined according to the ChapmanEnskog theory and the approach by Millington and Quirk (1961) which accounts for tortuosity due to porous matrix and water saturation (see Sect. A1). Table 4 compares theoretical with experimental effective binary diffusion coefficients of $\mathrm{CS}_{2}$ and argon in fine glass beads and Geba fine sand under the given experimental conditions (water saturation $S_{\mathrm{w}}$ and tortuosity $\tau$ ).

The experimental coefficients $D^{*}$ differed from the theoretical effective binary diffusion coefficient $D_{\mathrm{t}}^{*}$ calculated for the prevailing conditions. This could result from the choice of porous media, since both media were characterized by a uniform and narrow grain-size distribution, as well as the observed water-saturation profiles. Werner et al. (2004) reported that theoretical approaches are often sensitive since 


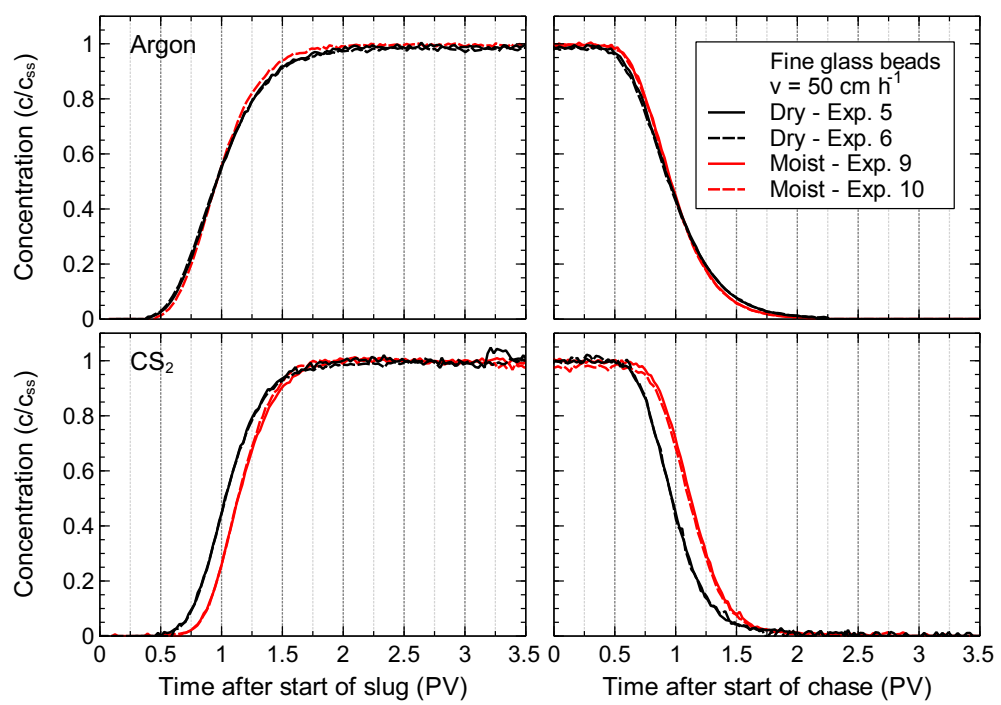

Figure 6. Breakthrough curves of $\mathrm{CS}_{2}$ and $\mathrm{Ar}$ in dry and moist $(\mathrm{Sw}=0.088)$ fine glass beads under identical slug and flow conditions $\left(v=50 \mathrm{~cm} \mathrm{~h}^{-1}\right)$.

the majority of their parameters are raised to a high power and do not apply satisfactorily to a wide variety of soils. Furthermore, the theoretical approach does not take into account material characteristics such as the pore-size distribution which may vary for similar porosities and hence affect transport. In addition, the equation used to determine the dispersion coefficient (Eq. A10) from TMA raises the velocity to the power of three; thus, minor deviations had a great impact on the final values.

The dispersivity $\alpha$ is a parameter of the porous medium only and should be independent of the components (gases) and flow conditions. A mean dispersivity of $\alpha_{\mathrm{GBfine}}=0.958 \mathrm{~cm}$ in fine glass beads and $\alpha_{\mathrm{Geba}}=0.432 \mathrm{~cm}$ in Geba fine sand was found. However, a difference between the dispersivity values of argon and $\mathrm{CS}_{2}$ in the same porous medium was found in the experiments (see Table 4), indicating that dispersivity is component dependent due to, e.g., diffusional or non-equilibrium effects. This component-dependent dispersivity has been shown by Costanza-Robinson and Brusseau (2002a), who reported that dispersivity ranges from approximately 0.1 to $5.0 \mathrm{~cm}$. Hence, greater reliability was attributed to the dispersivity $\alpha_{\mathrm{Ar}}$ determined from BTCs of argon since it is a conservative tracer.

\subsection{Retardation of $\mathrm{CS}_{2}$}

Different series of experiments were conducted to quantify retardation of $\mathrm{CS}_{2}$ as a function of water saturation and seepage velocity. Figures 6 and 7 compare BTCs of argon and $\mathrm{CS}_{2}$ in dry (black) and moist (red) porous medium for the same seepage velocity $\left(v=50 \mathrm{~cm} \mathrm{~h}^{-1}\right)$. Two to three repetitions of each run in dry and moist conditions, respectively, are shown. The graphs show normalized concentrations as a function of effective pore volume (total pore volume minus water content after drainage).

The BTCs of argon showed excellent reproducibility in repetition experiments in both materials with the same conditions $\left(v=50 \mathrm{~cm} \mathrm{~h}^{-1}\right)$. In fine glass beads, argon showed very similar BTCs for dry and moist experiments. Thus, this confirmed that argon experiences no retardation and may be used as a conservative tracer and as a reference for $\mathrm{CS}_{2}$. In Geba fine sand, a different skewness was observed between dry and moist conditions as a result of the reduced pore space in moist conditions. Hence, a comparison of the BTCs revealed that the effective-flow region in fine glass beads was similar in dry and moist conditions, whereas in Geba fine sand it was reduced in moist conditions. This resulted in BTCs which were less affected by diffusion due to a shorter residence time. Since the experiments were conducted with a constantflow-rate boundary condition based on the calculated effective pore volume, a shorter residence time, i.e., higher seepage velocity, occurred when the actual effective pore volume available for gas flow was smaller than the calculated volume.

The BTCs of $\mathrm{CS}_{2}$ showed, in general, good reproducibility for all experiments. In fine glass beads, a later breakthrough of $\mathrm{CS}_{2}$ compared to argon can be observed in Fig. 6, demonstrating the retardation of $\mathrm{CS}_{2}$ due to partitioning processes. The different effective pore volume due to water and possible reduced residence time (actual vs. calculated PV) resulted in less skewed BTCs compared to the dry experiments. In Geba fine sand, a more pronounced retardation of $\mathrm{CS}_{2}$ was observed compared to experiments in fine glass beads. The later breakthrough becomes evident when comparing BTCs in dry (black) with moist (red) conditions in Fig. 7. In two of the three BTCs in moist experiments (Fig. 7), $\mathrm{CS}_{2}$ con- 


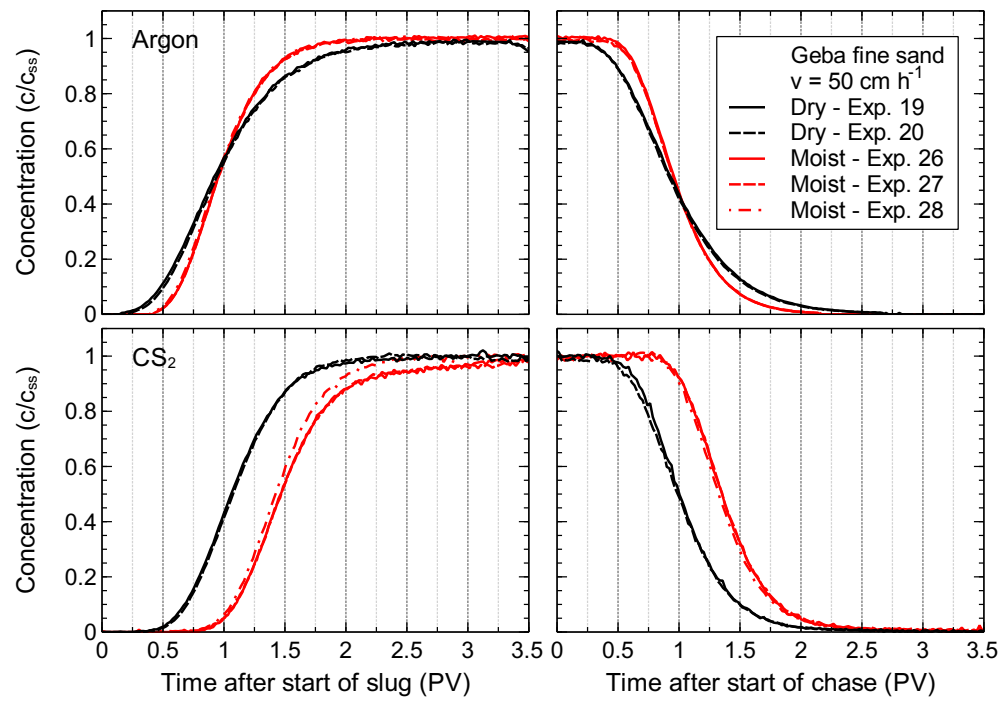

Figure 7. Breakthrough curves of $\mathrm{CS}_{2}$ and $\mathrm{Ar}$ in dry and moist $\left(S_{\mathrm{W}}=0.154\right)$ Geba fine sand under identical slug and flow conditions $\left(v=50 \mathrm{~cm} \mathrm{~h}^{-1}\right)$.

centrations leveled at around $c / c_{\mathrm{ss}}=0.9$ followed by an increase to steady-state (plateau) concentrations toward the end of the slug. This behavior might be a consequence of the water saturation over column height affecting the partitioning processes.

The retardation coefficients of $\mathrm{CS}_{2}$ as a function of porous medium, water saturation, and seepage velocity were determined using the TMA of the breakthrough curves (see Sect. 2.3). The coefficients were normalized with respect to the BTCs from dry porous medium. Thereby, errors due to setup or other systematic errors could be eliminated and allowed for the comparison with theoretical values. Figure 8 shows retardation coefficients of $\mathrm{CS}_{2}$ as a function of water saturation (upper) and seepage velocity (lower) in fine glass beads (black) and Geba fine sand (red). The coefficients of the slug (circle) and the chase (rectangle) are given. Note the broken $x$ axis (water saturation) between $S_{\mathrm{w}}=0.10$ and 0.13 in the upper graph indicated by the vertical dashed lines.

In fine glass beads, a nonlinear increase in the retardation coefficient from $R_{\mathrm{GBfine}}=1.09$ to 1.16 with increasing water saturation from $S_{\mathrm{w}}=0.075$ to 0.155 was observed. Of course, partitioning into the water phase and adsorption to the air-water interface depend on the interfacial area which should decrease with increasing water saturation. Thus, an extrapolation of the retardation coefficient to higher water saturations might be difficult. The retardation of the slug and of the chase was different in fine glass beads, the chase being more prone to retardation than the slug. The breakthrough of the nitrogen chase (removal of the $\mathrm{CS}_{2}$ vapor) showed a higher retardation by a factor (average) of 1.05 compared to the breakthrough of the slug throughout all experiments in fine glass beads. This behavior can be also seen when comparing the BTCs of $\mathrm{CS}_{2}$ in the upper graph of Fig. 6.
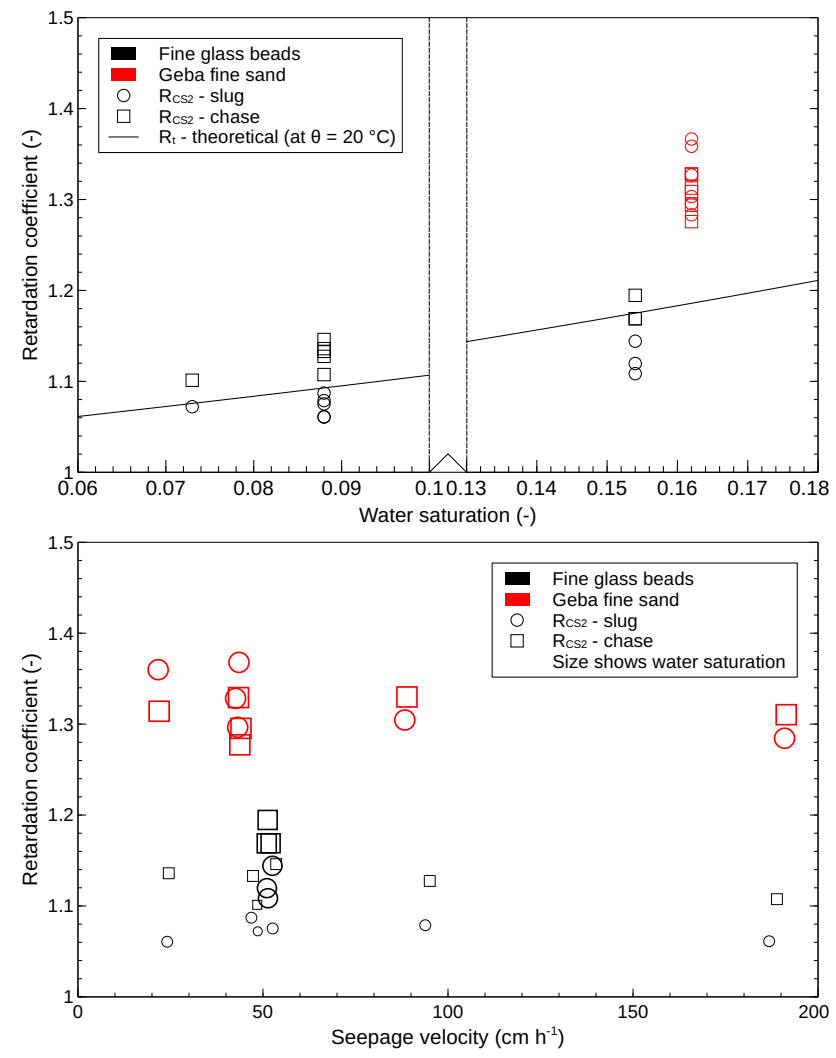

Figure 8. Retardation coefficients of $\mathrm{CS}_{2}$ determined from experiments with different seepage velocities in fine glass beads and in Geba fine sand at different water saturations (evaluated with temporal-moment analysis). 
In Geba fine sand, higher retardation coefficients compared to fine glass beads were measured in the experiments. These ranged between $R_{\text {Geba }}=1.29$ and 1.34 at a mean water saturation of $S_{\mathrm{W}}=0.162$. This was due to the higher water saturation and its increase toward the bottom (discussed in Sect. 3.1), the different gas-water interfacial area, and the pore space available for gas flow. Series 3 in Geba fine sand had to be excluded from these graphs due to mass balance issues discussed later. Hence, results were only available for one particular water-saturation profile in Geba fine sand. The ratio between the retardation coefficient of the slug and that of the chase did not show a clear trend as observed in fine glass beads despite the differences seen in Fig. 8.

An evaluation of the processes responsible for retardation is possible when utilizing the analytical solution (Eq. 2) proposed by Brusseau et al. (1997). The equation includes accumulation in the gas phase, partitioning into the aqueous phase, adsorption to the solid phase, and adsorption to the gas-water interface. Partitioning into the aqueous phase (dissolution) is controlled by the temperature- and componentdependent Henry constant. Since equilibrium is assumed, the relative contribution of this process to the total retardation is considered as maximum.

Adsorption to the solid phase is governed by the partitioning coefficient $K_{\mathrm{D}}$ of that particular component. $K_{\mathrm{D}}$ is the product of the soil organic carbon partitioning coefficient $K_{\mathrm{OC}}$ and the fraction of organic carbon in the soil material $f_{\text {OC }}$. According to the Superfund Soil Screening Guidance (US Environmental Protection Agency, 1996) for $\mathrm{CS}_{2}$, the coefficient $K_{\mathrm{OC}}$ is $45.7 \mathrm{~L} \mathrm{~kg}^{-1}$. Howard et al. (1990) reported that "carbon disulfide in solution would therefore not be expected to adsorb significantly to soil" due to the relatively low $K_{\mathrm{OC}}$. The two types of materials used, fine glass beads and Geba fine sand, were evaluated with respect to organic carbon. The chemical composition of fine glass beads (soda-lime glass) is given by the manufacturer (Sigmund Lindner GmbH, SiLibeads Type S 100-200 $\mu \mathrm{m})$ as $\mathrm{SiO}_{2}(72.5 \%), \mathrm{Na}_{2} \mathrm{O}(13 \%), \mathrm{CaO}(9.1 \%), \mathrm{MgO}(4.2 \%)$, and $\mathrm{Al}_{2} \mathrm{O}_{3}(0.58 \%)$. Geba fine sand (Quarzsande $\mathrm{GmbH}$, Geba weiss, 63-350 $\mu \mathrm{m})$ is composed of $\mathrm{SiO}_{2}(99.2 \%), \mathrm{Fe}_{2} \mathrm{O}_{3}$ $(0.09 \%), \mathrm{Al}_{2} \mathrm{O}_{3}(1.85 \%)$, and $\mathrm{TiO}_{2}(0.24 \%)$, and thus is a pure quartz sand. Both materials contain negligible fractions of organic carbon $\left(f_{\mathrm{OC}}\right)$; hence, adsorption of $\mathrm{CS}_{2}$ on the grains did not significantly contribute to retardation and may be neglected.

Adsorption on the air-water interface in a partially watersaturated porous medium depends on the air-water partitioning coefficient $K_{\mathrm{IA}}$ and on the air-water interfacial area $A_{\mathrm{IA}}$. The air-water partitioning coefficient $K_{\mathrm{IA}}$ of $\mathrm{CS}_{2}$ was estimated using the empirical correlation (Eq. 4 with Eq. 5) proposed by Valsaraj (1988). This correlation with $\log \left(K_{\text {Ow }}\right)=2.00$ and the dimensionless Henry constant $K_{\mathrm{H}}=1.04$ yielded the air-water partitioning coefficient of $\mathrm{CS}_{2} K_{\mathrm{IA}}=6.87 \times 10^{-6} \mathrm{~cm}$. Costanza-Robinson et al. (2008) found a correlation (Eq. 6) based on X-ray microtomogra- phy measurements of glass beads and natural sands to estimate the interfacial area. The calculated interfacial areas for a water-saturation range from 0.05 to 0.20 decreases from 19.5 to $16.4 \mathrm{~cm}^{-1}$ for fine glass beads and from 20.9 to $17.5 \mathrm{~cm}^{-1}$ for Geba fine sand.

The theoretical retardation coefficient (Eq. 2) as a function of water saturation was calculated based on the assumptions, correlations and parameters discussed above. It is shown as a line in the upper graph of Fig. 8. Only one line is plotted for both materials as the results are nearly identical due to similar porosities and calculated interfacial areas. In fine glass beads, the theoretical coefficient compared very well with the values from the experiments. It slightly overpredicted the retardation of the slug while it underpredicted that of the chase; however, it reproduced satisfactorily the mean retardation coefficient and its increase with water saturation. In Geba fine sand, the theoretical coefficient, taking into account dissolution and adsorption to the air-water interface, significantly underestimated the observed retardation. The ratio between the contributions from dissolution $\beta_{\mathrm{W}}$ and from air-water interfacial adsorption $\beta_{\mathrm{IA}}$ at $S_{\mathrm{W}}=0.162$ yields $\beta_{\mathrm{w}} / \beta_{\mathrm{IA}}=513$. Since dissolution, which is a function of the Henry constant and assumes equilibrium, is already at its maximum and adsorption to the grains may be neglected as discussed above, it is postulated that this increased retardation can only be caused by air-water interfacial adsorption. Possible reasons for an underestimation of the adsorption to the air-water interface could be that the inhomogeneous water-saturation profiles (see Fig. 4), the grainsize distribution, or the underestimation of the specific surface of the Geba fine sand particles led to an underestimation of the interfacial area. Scanning electron microscopy images (Fig. 3) suggested that the smooth-sphere assumption holds for glass beads but not necessarily for Geba fine sand; thus, the interfacial area for Geba fine sand might have been significantly underestimated. Interfacial areas measured with microtomography are smaller than those determined with vapor-phase tracer experiments (Brusseau et al., 2006; Costanza-Robinson et al., 2008). Furthermore, they reported that $A_{\text {IA }}$ from microtomography measurements approach a maximum value similar to the smooth-sphere assumption while measurements from vapor-phase experiments rather approach a maximum close to the specific surface area of the material (Brunauer-Emmett-Teller measurements) as water saturation approaches zero. Costanza et al. (2000) observed the maximum interfacial area for water saturation in the range of 15 to $25 \%$. They also reported the possibility of multilayer adsorption and that the actual adsorption may be significantly underestimated when true $A_{\text {IA }}$ values are used.

Attributing the observed discrepancy in Geba fine sand to air-water interfacial adsorption of $\mathrm{CS}_{2}$, an interfacial area of about $A_{\text {IA,calc }}=6553 \mathrm{~cm}^{-1}$ would be required to obtain a mean retardation factor of $R_{\mathrm{Geba}}=1.31$. This is consistent with measured interfacial areas from vapor-phase tracer experiments (e.g., Costanza et al., 2000). Further experiments 
would be required using an additional tracer for air-water interfacial adsorption to actually measure the area and thereby quantify the relative contribution of dissolution and interfacial adsorption to the total retardation of $\mathrm{CS}_{2}$. The findings suggest that retardation may vary with changing saturation as a function of distance from the groundwater table due to varying relative contributions of the partitioning processes. Since water saturation increases toward the groundwater table, more contaminant mass can partition into the bulk water phase but less mass is adsorbed to the air-water interface due to a reduced interfacial area.

The lower graph in Fig. 8 shows the retardation coefficients as a function of seepage velocity. A mean retardation coefficient of $R_{\mathrm{GB} f i n e}=1.100 \pm 0.0096$ and $R_{\text {Geba }}=1.315 \pm 0.0152$ was measured in the experiments with fine glass beads $\left(S_{\mathrm{w}}=0.088\right)$ and Geba fine sand $\left(S_{\mathrm{w}}=0.162\right)$, respectively. In general, no significant change of the retardation behavior with increasing seepage velocity was observed. This confirmed that the mass transport rate was low enough and the residence time of the slug was sufficient for the partitioning processes to reach equilibrium. Concluding from the experiment with $v=200 \mathrm{~cm} \mathrm{~h}^{-1}$, it seems likely that there was a slight tendency toward a reduced retardation. In fact, retardation may reduce at higher seepage velocities due to limiting contaminant diffusion. If no equilibrium is reached in the event of high velocities, the retardation coefficient reflects an apparent coefficient since in this case it is a function of the experimental system used (i.e., length of the column). Additional experimental repetitions would have been required to provide proof. However, this experimental investigation aimed at characterizing retardation of $\mathrm{CS}_{2}$ in the range of velocities observed during vapor-plume migration experiments $\left(v \ll 200 \mathrm{~cm} \mathrm{~h}^{-1}\right)$. Hence, the focus was laid on the velocities used and higher values were beyond the scope.

Mass balance analyses were performed to obtain mass recovery $(r)$ from each breakthrough curve. Mass recovery was calculated from concentration and flow measurements and was normalized with respect to the injected mass. In general, mass recoveries of argon and $\mathrm{CS}_{2}$ showed good results. Mean recovery calculated from all experiments conducted yielded for argon $r_{\mathrm{Ar}}=0.995 \pm 0.007$ and for $\mathrm{CS}_{2}$ $r_{\mathrm{CS}}^{2}=0.981 \pm 0.084$ (excluding experiments of Series 3 ). Mass recoveries of all experiments are given in Tables B1 and B2 in the Appendix. Complete mass recoveries proved reliability and quality of the results gained from these column experiments.

The results discussed above excluded Series 3 in Geba fine sand. Series 3 referred to the second saturation and drainage cycle which was carried out to establish a static water saturation different than in Series 2. However, significant $\mathrm{CS}_{2}$ mass losses were observed and became more pronounced with each experiment in this series, eventually leading to their exclusion from the results. Recoveries of $\mathrm{CS}_{2}$ decreased from $r_{\mathrm{CS}}^{2}=0.854$ in the first experiment of Series 3 down to
$r_{\mathrm{CS}}^{2}=0.010$. This mass loss of $\mathrm{CS}_{2}$ was caused by biodegradation which was confirmed by the smell of hydrogen sulfide in the column outflow. The mass balance analysis of the experiments enabled for determining mean degradation rates of $\mathrm{CS}_{2}$ which were calculated from the $\mathrm{CS}_{2}$ mass rate and the recovery. The mean degradation rates ranged from 0.12 to $1.28 \mathrm{mg} \mathrm{h}^{-1}$ depending on the $\mathrm{CS}_{2}$ mass flux applied in the experiments. Microbial degradation of $\mathrm{CS}_{2}$ under aerobic and anaerobic conditions has been studied in the field of waste-gas treatment (e.g., Smith and Kelly, 1988; Hartikainen et al., 2000; Pol et al., 2007). They found microbes of the Thiobacillus species able to oxidize $\mathrm{CS}_{2}$ under certain conditions. Cox et al. (2013) were the first to study degradation in soils and groundwater from a $\mathrm{CS}_{2}$-contaminated site. They confirmed carbonyl sulfide (COS) and hydrogen sulfide $\left(\mathrm{H}_{2} \mathrm{~S}\right)$ as byproducts and reported almost $100 \% \mathrm{CS}_{2}$ degradation due to biological activity in their soil experiments. However, they also found evidence for microbial selfinhibition with increasing $\mathrm{CS}_{2}$ concentration. This has been also reported by Pol et al. (2007) who additionally found inhibition of degradation due to accumulation of the intermediate products, $\mathrm{COS}$ and $\mathrm{H}_{2} \mathrm{~S}$. Hence, biodegradation is only relevant in specific environments and conditions and is most likely reduced as concentrations increase closer to the source zone of a contaminated site. This may explain why $\mathrm{CS}_{2}$ is still persistent at many of the NPL sites listing $\mathrm{CS}_{2}$ as a contaminant of concern.

A direct comparison with reported degradation rate constants determined from batch experiments could not be achieved due to the only availability of effluent gas concentrations in our experiments. Nonetheless, they showed that biodegradation may have a considerable potential for mitigating the contaminant mass transfer by vapor migration to the underlying aquifer, provided that favorable conditions for the specific microbes can be ensured, for instance, via soil venting. A detailed investigation of biodegradation was beyond the scope of this work but should be addressed in future research.

\section{Conclusions}

- The retardation of $\mathrm{CS}_{2}$ vapor was quantified in $2 \mathrm{~m}$ long column experiments in two different porous media as a function of water saturation and velocity by comparison with the conservative tracer argon. The temporalmoment analysis for a step input (TMA) was applied to determine retardation factors and dispersion coefficients from concentration breakthrough curves. Comparison between experiments was achieved by relating mean arrival times of $\mathrm{CS}_{2}$ to those of argon. The retardation coefficient of $\mathrm{CS}_{2}$ increased from around 1.08 to 1.14 with increasing water saturation from 0.073 to 0.154 in fine glass beads. A slightly higher retardation of the chase by a factor of 1.05 compared to that of the slug was ob- 
served. A pronounced higher mean retardation of 1.31 was observed in Geba fine sand and a water saturation of 0.162 due to the different grain-size distribution and the particular water-saturation profile. Retardation coefficients as a function of (seepage) velocity revealed only a minor dependency and suggested a slight tendency toward a reduced retardation at higher velocities.

- The experimental retardation coefficients were compared to an analytical solution considering accumulation in the gas phase, partitioning to the aqueous phase, and adsorption to the air-water interface. Adsorption to the solid phase was neglected due to negligible fractions of organic matter in the porous media used. The analytic solution compared very well with the experimental results in fine glass beads, identifying dissolution as the main contribution to retardation. However, it underpredicted retardation in Geba fine sand. The discrepancy was ascribed to an increased relative contribution of airwater interfacial adsorption in Geba fine sand as a result of a significant underestimation of the interfacial area. They were estimated using a correlation derived from microtomography measurements of glass beads and natural soils and utilizing the smooth-sphere assumption (Costanza-Robinson et al., 2008). A readily applicable correlation to estimate interfacial areas is still considered a challenge and vapor-phase tracer experiments are so far the only reliable method to measure effective interfacial areas for vapor retardation. A quantification of the effective interfacial area could be achieved by using additional tracers which predominantly adsorb to the air-water interface.
- Clear evidence of the biodegradation of $\mathrm{CS}_{2}$ was found in the last series of experiments in Geba fine sand confirmed by the concentration measurements and the mass balance analysis. These findings demonstrate the potential of biodegradation to reduce the total $\mathrm{CS}_{2}$ mass of migrating vapor plumes eventually threatening the underlying aquifer. Biodegradation of $\mathrm{CS}_{2}$ has been confirmed by several experimental studies (e.g., Pol et al., 2007; Cox et al., 2013); however, they showed that $\mathrm{CS}_{2}$ degradation is only relevant in specific environments and conditions due to microbial self-inhibition at higher concentrations. Thus, site-specific investigations are needed to evaluate the potential for biodegradation or natural attenuation of a $\mathrm{CS}_{2}$ contamination. Soil venting could be used to reduce self-inhibition and enhance biodegradation. The experiments showed a complete removal of the $\mathrm{CS}_{2}$ vapor and a reversibility of partitioning processes confirmed by mass balance analyses. Vaporization rates of liquid $\mathrm{CS}_{2}$ were not studied in this work and need to be investigated to estimate the removal efficiency of a liquid spill. Nevertheless, a fast vaporization of liquid $\mathrm{CS}_{2}$ is expected due to its low boiling point and high vapor pressure. This clearly supports the remediation of a $\mathrm{CS}_{2}$ contamination in the unsaturated zone using soil-vapor extraction.

\section{Data availability}

The experimental data used to produce the results and graphs presented in this paper are available at doi:10.4228/ZALF.2013.298 (Kleinknecht, 2016). 


\section{Appendix A: Materials and methods}

\section{A1 Binary diffusion coefficient}

The Chapman-Enskog formula is used to estimate the binary diffusion coefficient of component $A$ in $B$ at low density.

$D_{A B}=1.8583 \times 10^{-3} \frac{\sqrt{T^{3}\left(\frac{1}{M_{A}}+\frac{1}{M_{B}}\right)}}{p \sigma_{A B}^{2} \Omega_{D, A B}}$,

with $D_{A B}\left(\mathrm{~cm}^{2} \mathrm{~s}^{-1}\right)$, temperature $T(\mathrm{~K})$, pressure $p(\mathrm{~atm})$, the Lennard-Jones parameter $\sigma_{A B}$ (angstrom), and the collision integral $\Omega_{D, A B}$ which can be approximated with the Lennard-Jones potential. Component-specific values to determine $\sigma_{A B}$ as well as $\Omega_{D, A B}$ as a function of $k T / \epsilon$ can be found in Bird et al. (1960).

Porous media affect diffusion of gases since space is occupied by grains and possibly by additional fluid phases. Therefore, Fick's law is often modified by the factor $\beta$ to account for these deviations.

$D^{*}=\beta D_{A B}$,

while $\beta$ is defined as

$\beta=\phi S_{g} \tau$,

where $D_{A B}^{*}$ is the effective diffusion coefficient in porous media, $\phi$ is the porosity, $S_{\mathrm{g}}$ the gas saturation (equal to 1 for the all-gas condition), and $\tau$ is the tortuosity. According to Millington and Quirk (1961), tortuosity can be approximated by

$\tau=\phi^{1 / 3} S_{\mathrm{g}}^{7 / 3}$.

\section{A2 Dispersion coefficient}

Flow of fluids in a porous medium may vary significantly on a microscale due to the velocity field in pores, irregularities of the pore size, flow restrictions, or dead-end pores resulting in additional spreading denoted as dispersion. These influences have to be taken into account in analytical or numerical solutions of flow in porous media. This is done by introducing the longitudinal dispersion coefficient

$D=\beta D_{A B}+\alpha v=D^{*}+\alpha v$,

with the dispersion coefficient $D\left(\mathrm{~cm}^{2} \mathrm{~s}^{-1}\right)$, effective binary diffusion coefficient $D_{A B}^{*}\left(\mathrm{~cm}^{2} \mathrm{~s}^{-1}\right)$ according to Eq. (A2), gas-phase longitudinal dispersivity $\alpha(\mathrm{cm})$, and average gas velocity $v\left(\mathrm{~cm} \mathrm{~s}^{-1}\right)$.

\section{A3 Temporal-moment analysis}

The measured BTC data had to be prepared to allow for the usage of the TMA generally applied to responses from dirac input. The breakthrough curves of the step-input boundary condition (1) were transformed to a dirac-input boundary condition (2) (Yu et al., 1999). This was achieved by using the derivative of the original step-input BTC data.

$\frac{\partial c_{1}}{\partial t}=c_{2}$

This transformation then allowed for analyzing the original breakthrough curves and required adapted definitions of the temporal moments. The first-order normalized moment $M_{1}$ representing the mean breakthrough arrival time $(\tau)$ is then defined as

$\tau=M_{1}=\frac{m_{1}}{m_{0}}=\frac{\int_{0}^{1} t d c_{1}}{\int_{0}^{1} d c_{1}}$,

where $c_{1}(-)$ is normalized concentration of measured BTC and $t$ (second or PV) is elapsed time. The second central moment $\mu_{2}$ corresponds to the variance of travel times at the location of measurement and is given by

$\mu_{2}=\int_{0}^{1}\left(t-M_{1}\right)^{2} d c_{1}$.

These two moments can be used to directly infer seepage velocity $v$ and dispersion coefficient $D$ from BTC data for a one-dimensional system (Cirpka and Kitanidis, 2000).

$v=\frac{z}{M_{1}}$

$D=\frac{\mu_{2} v^{3}}{2 z}$

\section{Appendix B: Detailed experimental results}

Tables B1 and B2 show the theoretical seepage velocity, the injection duration (slug), the injected mass, and the normalized recovery of the components $\mathrm{CS}_{2}$ and argon. The tables list all experiments in order according to the conducted series. 
Table B1. Experimental conditions of vapor-retardation experiments in fine glass beads: series, experiment, theoretical seepage velocity, injection duration, and injected mass and recovery of $\mathrm{CS}_{2}$ and argon.

\begin{tabular}{cccccccc}
\hline $\begin{array}{c}\text { Series } \\
\text { no. }\end{array}$ & $\begin{array}{c}\text { Exp. } \\
\text { no. }\end{array}$ & $\begin{array}{c}v \\
\mathrm{~cm} \mathrm{~h}^{-1}\end{array}$ & $\begin{array}{c}t_{\mathrm{inj}} \\
\mathrm{h}\end{array}$ & $\begin{array}{c}m_{\mathrm{Ar}} \\
\mathrm{mg}\end{array}$ & $\begin{array}{c}m_{\mathrm{CS} 2} \\
\mathrm{mg}\end{array}$ & $\begin{array}{c}r_{\mathrm{Ar}} \\
-\end{array}$ & $\begin{array}{c}r_{\mathrm{CS} 2} \\
-\end{array}$ \\
\hline 1 & 1 & 25 & 27.80 & 667.8 & 2671.4 & 0.994 & 1.123 \\
& 2 & 50 & 13.92 & 677.7 & 2710.9 & 1.011 & 1.066 \\
& 3 & 50 & 14.10 & 675.9 & 2703.5 & 0.996 & 1.005 \\
& 4 & 50 & 13.65 & 677.7 & 542.2 & 0.992 & 1.033 \\
& 5 & 50 & 14.29 & 690.3 & 13.8 & 0.988 & 0.822 \\
& 6 & 50 & 14.07 & 687.0 & 13.7 & 0.984 & 0.753 \\
& 7 & 50 & 13.82 & 673.3 & 13.5 & 0.987 & 0.770 \\
\hline 2 & 8 & 25 & 28.35 & 619.9 & 12.4 & 0.989 & 1.022 \\
& 9 & 50 & 14.40 & 617.8 & 12.4 & 0.993 & 0.983 \\
& 10 & 50 & 12.83 & 615.2 & 12.3 & 0.997 & 1.010 \\
& 11 & 100 & 7.22 & 620.8 & 12.4 & 1.000 & 0.941 \\
& 12 & 200 & 3.56 & 617.1 & 12.3 & 0.998 & 0.941 \\
\hline 3 & 13 & 50 & 14.38 & 635.0 & 12.7 & 0.995 & 1.054 \\
& 14 & 50 & 14.22 & 634.0 & 12.7 & 1.000 & 1.032 \\
& 15 & 50 & 14.43 & 635.4 & 12.7 & 0.989 & 1.013 \\
\hline 4 & 16 & 50 & 14.06 & 626.0 & 12.5 & 0.994 & 1.039 \\
\hline
\end{tabular}

Table B2. Experimental conditions of vapor-retardation experiments in Geba fine sand: series, experiment, theoretical seepage velocity, injection duration, and injected mass and recovery of $\mathrm{CS}_{2}$ and argon.

\begin{tabular}{|c|c|c|c|c|c|c|c|}
\hline $\begin{array}{c}\text { Series } \\
\text { no. }\end{array}$ & $\begin{array}{c}\text { Exp. } \\
\text { no. }\end{array}$ & $\begin{array}{c}v \\
\mathrm{~cm} \mathrm{~h}^{-1}\end{array}$ & $\begin{array}{c}t_{\text {inj }} \\
\mathrm{h}\end{array}$ & $\begin{array}{c}m_{\mathrm{Ar}} \\
\mathrm{mg}\end{array}$ & $\begin{array}{c}m_{\mathrm{CS} 2} \\
\mathrm{mg}\end{array}$ & $\begin{array}{c}r \mathrm{Ar} \\
-\end{array}$ & $\begin{array}{c}r \mathrm{CS} 2 \\
-\end{array}$ \\
\hline \multicolumn{8}{|c|}{ Geba fine sand } \\
\hline \multirow[t]{8}{*}{1} & 17 & 25 & 28.71 & 673.9 & 13.5 & 0.982 & 0.964 \\
\hline & 18 & 25 & 40.97 & 944.5 & 18.9 & 0.992 & 0.942 \\
\hline & 19 & 50 & 14.30 & 599.2 & 12.0 & 0.985 & 1.006 \\
\hline & 20 & 50 & 14.31 & 597.5 & 12.0 & 0.983 & 0.970 \\
\hline & 21 & 100 & 7.20 & 664.8 & 13.3 & 0.999 & 0.994 \\
\hline & 22 & 100 & 7.32 & 665.9 & 13.3 & 0.999 & 1.062 \\
\hline & 23 & 200 & 3.83 & 706.2 & 14.1 & 1.000 & 0.994 \\
\hline & 24 & 200 & 3.54 & 657.7 & 13.2 & 0.995 & 0.962 \\
\hline \multirow[t]{6}{*}{2} & 25 & 25 & 41.17 & 795.7 & 15.9 & 0.984 & 1.009 \\
\hline & 26 & 50 & 14.33 & 552.8 & 11.1 & 1.000 & 0.955 \\
\hline & 27 & 50 & 20.28 & 767.3 & 15.3 & 0.992 & 1.115 \\
\hline & 28 & 50 & 20.62 & 799.5 & 16.0 & 0.997 & 0.973 \\
\hline & 29 & 100 & 10.12 & 797.6 & 16.0 & 0.987 & 0.965 \\
\hline & 30 & 200 & 4.67 & 800.8 & 16.0 & 1.000 & 1.013 \\
\hline \multirow[t]{7}{*}{3} & 31 & 50 & 20.54 & 815.8 & 16.3 & 0.994 & 0.854 \\
\hline & 32 & 50 & 24.04 & 955.8 & 19.1 & 0.998 & 0.684 \\
\hline & 33 & 100 & 11.02 & 864.1 & 17.3 & 1.000 & 0.534 \\
\hline & 34 & 200 & 5.16 & 782.9 & 15.7 & 1.008 & 0.689 \\
\hline & 35 & 25 & 49.26 & 957.3 & 19.1 & 1.012 & 0.010 \\
\hline & 36 & 100 & 10.28 & 793.0 & 15.9 & 1.000 & 0.174 \\
\hline & 37 & 50 & 23.81 & 944.0 & 18.9 & 0.996 & 0.016 \\
\hline
\end{tabular}


Author contributions. Simon M. Kleinknecht designed and conducted this experimental study. Holger Class and Jürgen Braun were responsible for the scientific and experimental supervision. Simon M. Kleinknecht prepared the paper with contributions from both co-authors.

Competing interests. The authors declare that they have no conflict of interest.

Acknowledgements. The authors thank the reviewers whose insightful comments and suggestions improved the manuscript.

Edited by: B. Berkowitz

Reviewed by: two anonymous referees

\section{References}

Barber, C. and Davis, G. B.: Fugacity model to assess the importance of factors controlling the movement of volatile organics from soil to ground water, in: Proceedings of a Workshop on Modelling the Fate of Chemicals in the Environment, The Australian National University, Centre for Resource and Environmental Studies, Water Research Foundation of Australia, Canberra, ACT, 67-73, 1991.

Bird, R. B., Stewart, W. E., and Lightfoot, E. N.: Transport phenomena, Wiley, New York, 1960.

Brusseau, M. L., Popovičová, J., and Silva, J. A. K.: Characterizing gas-water interfacial and bulk-water partitioning for gas-phase transport of organic contaminants in unsaturated porous media, Environ. Sci. Technol., 31, 1645-1649, doi:10.1021/es960475j, 1997.

Brusseau, M. L., Peng, S., Schnaar, G., and Costanza-Robinson, M. S.: Relationships among air-water interfacial area, capillary pressure, and water saturation for a sandy porous medium, Water Resources Research, 42, w03501, doi:10.1029/2005WR004058, 2006.

Brusseau, M. L., Carroll, K. C., Truex, M. J., and Becker, D. J.: Characterization and Remediation of Chlorinated Volatile Organic Contaminants in the Vadose Zone, Vadose Zone J., 12, doi:10.2136/vzj2012.0137, 2013.

Brusseau, M. L., Ouni, A. E., Araujo, J. B., and Zhong, H.: Novel methods for measuring air-water interfacial area in unsaturated porous media, Chemosphere, 127, 208-213, doi:10.1016/j.chemosphere.2015.01.029, 2015.

Budavari, S. (Ed.): The Merck index: an encyclopedia of chemicals, drugs, and biologicals, 12th Edn., Merck, Whitehouse Station, NJ, 1996.

Cabbar, H. and Bostanci, A.: Moisture effect on the transport of organic vapors in sand, J. Hazard. Mater., 82, 313-322, doi:10.1016/S0304-3894(01)00177-7, 2001.

Cirpka, O. A. and Kitanidis, P. K.: Characterization of mixing and dilution in heterogeneous aquifers by means of local temporal moments, Water Resour. Res., 36, 1221-1236, doi:10.1029/1999WR900354, 2000.
Corley, T. L., Farrell, J., Hong, B., and Conklin, M. H.: VOC accumulation and pore filling in unsaturated porous media, Environ. Sci. Technol., 30, 2884-2891, doi:10.1021/es950644k, 1996.

Costanza, M. S., and Brusseau, M. L.: Contaminant Vapor Adsorption at the Gas-Water Interface in Soils, Environ. Sci. Technol. 34, 1-11, doi:10.1021/es9904585, 2000.

Costanza-Robinson, M. S. and Brusseau, M. L.: Gas phase advection and dispersion in unsaturated porous media, Water Resou. Res., 38, 7-1-7-9, doi:10.1029/2001WR000895, 2002a.

Costanza-Robinson, M. S. and Brusseau, M. L.: Air-water interfacial areas in unsaturated soils: Evaluation of interfacial domains, Water Resour. Res., 38, 13-1-13-17, doi:10.1029/2001WR000738, 2002b.

Costanza-Robinson, M. S., Harrold, K. H., and Lieb-Lappen, R. M.: X-ray Microtomography Determination of Air-Water Interfacial Area-Water Saturation Relationships in Sandy Porous Media, Environ. Sci. Technol., 42, 2949-2956, doi:10.1021/es072080d, 2008.

Costanza-Robinson, M. S., Carlson, T. D., and Brusseau, M. L.: Vapor-phase transport of trichloroethene in an intermediate-scale vadose-zone system: Retention processes and tracer-based prediction, J. Contam. Hydrol., 145, 82-89, doi:10.1016/j.jconhyd.2012.12.004, 2013.

Cox, S. F., McKinley, J. D., Ferguson, A. S., O'Sullivan, G., and Kalin, R. M.: Degradation of carbon disulphide $\left(\mathrm{CS}_{2}\right)$ in soils and groundwater from a $\mathrm{CS}_{2}$-contaminated site, Environ. Earth Sci., 68, 1935-1944, doi:10.1007/s12665-012-1881-y, 2013.

Davis, A., Fennemore, G., Peck, C., Walker, C., McIlwraith, J., and Thomas, S.: Degradation of carbon tetrachloride in a reducing groundwater environment: implications for natural attenuation, Appl. Geochem., 18, 503-525, doi:10.1016/S08832927(02)00102-6, 2003.

Davis, G. B., Rayner, J. L., Trefry, M. G., Fisher, S. J., and Patterson, B. M.: Measurement and modeling of temporal variations in hydrocarbon vapor behavior in a layered soil profile, Vadose Zone J., 4, 225-239, doi:10.2136/vzj2004.0029, 2005.

Davis, G. B., Patterson, B., and Trefry, M.: Evidence for Instantaneous Oxygen-Limited Biodegradation of Petroleum Hydrocarbon Vapors in the Subsurface, Ground Water Monit. Remedia., 29, 126-137, doi:10.1111/j.1745-6592.2008.01221.x, 2009.

Goss, K.-U.: Predicting Adsorption of Organic Chemicals at the Air-Water Interface, J. Phys. Chem.-A, 113, 12256-12259, doi:10.1021/jp907347p, 2009.

Hartikainen, T., Ruuskanen, J., Räty, K., von Wright, A., and Martikainen, P.: Physiology and taxonomy of Thiobacillus strain TJ330, which oxidizes carbon disulphide $\left(\mathrm{CS}_{2}\right)$, J. Appl. Microbiol., 89, 580-586, doi:10.1046/j.1365-2672.2000.01150.x, 2000.

Höhener, P., Dakhel, N., Christophersen, M., Broholm, M., and Kjeldsen, P.: Biodegradation of hydrocarbons vapors: comparison of laboratory studies and field investigations in the vadose zone at the emplaced fuel source experiment, Airbase Værløse, Denmark, J. Contam. Hydrol., 88, 337-358, doi:10.1016/j.jconhyd.2006.07.007, 2006.

Howard, P., Sage, G., Jarvis, W., and Gray, D.: Handbook of environmental fate and exposure data for organic chemicals, in: Vol. II: Solvents, Lewis Publishers, Inc., Chelsea, MI, USA, 1990. 
Kibbey, T. C. G. and Chen, L.: A pore network model study of the fluid-fluid interfacial areas measured by dynamic-interface tracer depletion and miscible displacement water phase advective tracer methods, Water Resour. Res., 48, w10519, doi:10.1029/2012WR011862, 2012.

Kim, H., Annable, M. D., and Rao, P. S. C.: Influence of air-water interfacial adsorption and gas-phase partitioning on the transport of organic chemicals in unsaturated porous media, Environ. Sci. Technol., 32, 1253-1259, doi:10.1021/es970868y, 1998.

Kleinknecht, S. M.: Data sets of experimental study on retardation of a heavy NAPL vapor in partially saturated porous media, Leibniz-Zentrum für Agrarlandschaftsforschung (ZALF) e.V., Open Research Data, Müncheberg, doi:10.4228/ZALF.2013.298, 2016.

Kleinknecht, S. M., Class, H., and Braun, J.: Density-driven migration of heavy NAPL vapor in the unsaturated zone, Vadose Zone J., 14, doi:10.2136/vzj2014.12.0173, 2015.

Lagioia, R., Sanzeni, A., and Colleselli, F.: Air, water and vacuum pluviation of sand specimens for the triaxial apparatus, Soils Found., 46, 61-67, doi:10.3208/sandf.46.61, 2006.

Lide, D. R.: CRC Handbook of chemistry and physics, 86th Edn., CRC Press, Boca Raton, FL, 2005.

Luo, J., Cirpka, O. A., and Kitanidis, P. K.: Temporalmoment matching for truncated breakthrough curves for step or step-pulse injection, Adv. Water Resour., 29, 1306-1313, doi:10.1016/j.advwatres.2005.10.005, 2006.

Maxfield, B. T., Ginosar, D. M., McMurtrey, R. D., Rollins, H. W., and Shook, G. M.: The effect of moisture content on retention of fluorocarbon tracers on sand, Geothermics, 34, 47-60, doi:10.1016/j.geothermics.2004.06.003, 2005.

Mayes, M., Jardine, P., Mehlhorn, T., Bjornstad, B., Ladd, J., and Zachara, J.: Transport of multiple tracers in variably saturated humid region structured soils and semi-arid region laminated sediments, J. Hydrol., 275, 141-161, doi:10.1016/S00221694(03)00039-8, 2003.

Millington, R. J. and Quirk, J. P.: Permeability of porous solids, T. Faraday Soc., 57, 1200-1207, doi:10.1039/TF9615701200, 1961.

Pol, A., van der Drift, C., and Op den Camp, H.: Isolation of a carbon disulfide utilizing Thiomonas sp. and its application in a biotrickling filter, Appl. Microbiol. Biotechnol., 74, 439-446, doi:10.1007/s00253-006-0663-4, 2007.

Popovičová, J. and Brusseau, M. L.: Contaminant mass transfer during gas-phase transport in unsaturated porous media, Water Resour. Res., 34, 83-92, doi:10.1029/97WR02945, 1998.
Rad, N. and Tumay, M.: Factors affecting sand specimen fabrication by raining, Geotech. Test. J., 10, 31-37, doi:10.1520/GTJ10136J, 1987.

Riddick, J. A., Bunger, W. B., and Sakano, T. K.: Organic Solvents: Physical Properties And Methods Of Purification, in: vol. XV, 4th Edn., Wiley, New York, http://www.gbv.de/dms/ilmenau/toc/ 01792670X.PDF (last access: March 2017), 1986.

Rivett, M. O., Wealthall, G. P., Dearden, R. A., and McAlary, T. A.: Review of unsaturated-zone transport and attenuation of volatile organic compound (VOC) plumes leached from shallow source zones, J. Contam. Hydrol., 123, 130-156, doi:10.1016/j.jconhyd.2010.12.013, 2011.

Smith, N. A. and Kelly, D. P.: Oxidation of Carbon Disulphide as the Sole Source of Energy for the Autotrophic Growth of Thiobacillus thioparus Strain TK-m, Microbiology, 134, 30413048, doi:10.1099/00221287-134-11-3041, 1988.

Toride, N., Inoue, M., and Leij, F. J.: Hydrodynamic dispersion in an unsaturated dune sand, Soil Sci. Soc. Am. J., 67, 703-712, doi:10.2136/sssaj2003.0703, 2003.

US Agency for Toxic Substances and Disease Registry: Priority List of Hazardous Substance, https://www.atsdr.cdc.gov/spl/ (last access: 22 December 2016), 2015.

US Environmental Protection Agency: Superfund Soil Screening Guidance - Technical Background Document - Part 5: Chemical-Specific Parameters, Tech. rep., Office of Solid Waste and Emergency Response, https://semspub.epa.gov/work/HQ/ 175235.pdf (last access: March 2017), 1996.

US Environmental Protection Agency: National Priority List (NPL): advanced query form, https://cumulis.epa.gov/ supercpad/cursites/srchsites.cfm (last access: March 2017), 2016.

Valsaraj, K. T.: On the physico-chemical aspects of partitioning of non-polar hydrophobic organics at the air-water interface, Chemosphere, 17, 875-887, doi:10.1016/0045-6535(88)900604, 1988.

Werner, D., Grathwohl, P., and Höhener, P.: Review of field methods for the determination of the tortuosity and effective gas-phase diffusivity in the vadose zone, Vadose Zone J., 3, 1240-1248, doi:10.2136/vzj2004.1240, 2004.

Yu, C., Warrick, A. W., and Conklin, M. H.: A moment method for analyzing breakthrough curves of step inputs, Water Resour. Res., 35, 3567-3572, doi:10.1029/1999WR900225, 1999. 